\title{
Magnetic properties and field-driven dynamics of chiral domain walls in epitaxial $\mathbf{P t} / \mathbf{C o} / \mathrm{Au}_{x} \mathbf{P t}_{1-x}$ trilayers
}

\author{
Kowsar Shahbazi, ${ }^{1}$ Aleš Hrabec, ${ }^{1,2, *}$ Simone Moretti, ${ }^{3,4}$ Michael B. Ward,${ }^{5}$ Thomas A. Moore, ${ }^{1}$ Vincent Jeudy, \\ Eduardo Martinez, ${ }^{3}$ and Christopher H. Marrows ${ }^{1, \dagger}$ \\ ${ }^{1}$ School of Physics and Astronomy, University of Leeds, Leeds LS2 9JT, United Kingdom \\ ${ }^{2}$ Laboratoire de Physique des Solides, CNRS, Université Paris-Sud, Université Paris-Saclay, 91405 Orsay Cedex, France \\ ${ }^{3}$ Departamento Fisica Aplicada, University of Salamanca, Plaza de los Caidos s/n E-37008, Salamanca, Spain \\ ${ }^{4}$ Fachbereich Physik, Universität Konstanz, 78457 Konstanz, Germany \\ ${ }^{5}$ Leeds Electron Microscopy and Spectroscopy Centre, School of Chemical and Process Engineering, \\ University of Leeds, Leeds LS2 9JT, United Kingdom
}

(Received 11 September 2018; revised manuscript received 8 November 2018; published 10 December 2018)

\begin{abstract}
Chiral domain walls in ultrathin perpendicularly magnetized layers have a Néel structure stabilized by a Dzyaloshinskii-Moriya interaction (DMI) that is generated at the interface between the ferromagnet and a heavy metal. Different interface materials or properties are required above and below a ferromagnetic film in order to generate the structural inversion asymmetry needed to ensure that the DMI arising at the two interfaces does not cancel. Here we report on the magnetic properties of epitaxial $\mathrm{Pt} / \mathrm{Co} / \mathrm{Au}_{x} \mathrm{Pt}_{1-x}$ trilayers grown by sputtering onto sapphire substrates with $0.6 \mathrm{~nm}$ thick Co. As $x$ rises from 0 to 1 , a structural inversion asymmetry is progressively generated. We characterize the epilayer structure with $\mathrm{x}$-ray diffraction and cross-sectional transmission electron microscopy, revealing (111) stacking. The saturation magnetization falls as the proximity magnetization in $\mathrm{Pt}$ is reduced, whilst the perpendicular magnetic anisotropy $K_{\mathrm{u}}$ rises. The micromagnetic DMI strength $D$ was determined using the bubble expansion technique and also rises from a negligible value when $x=0$ to $\sim 1$ $\mathrm{mJ} / \mathrm{m}^{2}$ for $x=1$. The depinning field at which field-driven domain wall motion crosses from the creep to the depinning regime rises from $\sim 40$ to $\sim 70 \mathrm{mT}$, attributed to greater spatial fluctuations of the domain wall energy with increasing $\mathrm{Au}$ concentration. Meanwhile, the increase in DMI causes the Walker field to rise from $\sim 10$ to $\sim 280 \mathrm{mT}$, meaning that only in the $x=1$ sample is the steady flow regime accessible. The full dependence of domain wall velocity on driving field bears little resemblance to the prediction of a simple one-dimensional model, but can be described very well using micromagnetic simulations with a realistic model of disorder. These reveal a rise in Gilbert damping as $x$ increases.
\end{abstract}

DOI: 10.1103/PhysRevB.98.214413

\section{INTRODUCTION}

Research into magnetic thin films has made huge progress over the last few decades, largely due to the additional functionality emerging at interfaces between a magnetic film of few or sub-nanometer thickness and its surroundings. In addition to a wide range of novel magnetotransport effects [1], the magnetic properties are profoundly affected by the choice of materials with which those interfaces are made and the nature and quality of the interfaces. Emergent properties span both the statics and dynamics of the magnetization. One of the first to be discovered was the perpendicular magnetic anisotropy (PMA) [2], which can be strong enough to cause a spin-reorientation transition and lift the magnetization out of the film plane in the ground state. Forming an interface with a metal close to satisfying the Stoner criterion can lead to proximity magnetism [3], which can account for a significant proportion of the total moment if the magnetic layer is very

*Present address: Department of Materials, ETH Zürich, 8093 Zürich, Switzerland.

†c.h.marrows@leeds.ac.uk thin. Long-predicted [4], but only recently observed [5], is the interfacial Dzyaloshinskii-Moriya interaction (DMI), which arises due to inversion symmetry breaking and favors chiral magnetic states [6,7]. Meanwhile, the damping of magnetization dynamics can be enhanced by spin-pumping into the surrounding layers [8].

Magnetizing a film perpendicular to its plane is a highly demagnetizing configuration, and domain structures [9], separated by domain walls (DWs), commonly occur. Whilst magnetostatics alone favors a Bloch wall configuration, the interfacial DMI takes a form that would prefer a Néel structure with a fixed chirality [10]. This is important for the latest forms of racetrack memory that exploit PMA materials [11], since the DWs are driven by spin-orbit torques [12] that require a component of magnetization collinear to the electron flow. This is not trivial to arrange by other means and it explains, for example, why Bloch walls are insensitive to this type of torque [13], and why the wall chirality determines the direction of wall motion under current $[14,15]$.

Many of these effects, for instance, the PMA and DMI, require strong spin-orbit coupling (SOC) and so it is heavy metals that are commonly used to form the interfaces with the ultrathin magnetic layer, usually in polycrystalline multilayers 
prepared by sputtering. Nevertheless, properties such as the DMI are extremely sensitive to the interface quality $[16,17]$. To avoid such ambiguities and in order to explore this systematically, one has to grow such materials in a controlled way. Here, we experimentally investigate epitaxial layers of $\mathrm{Pt} / \mathrm{Co} / \mathrm{Au}_{x} \mathrm{Pt}_{1-x}$ prepared by sputtering onto single-crystal sapphire substrates [18]. Pt is a common pairing with Co that has strong PMA and DMI [16], and is close to satisfying the Stoner criterion [19], giving strong proximity magnetism. The choice of $\mathrm{Au}$, a heavy element with a fully filled $5 d$ band, is motivated by a negligible proximity effect $[15,20]$ and the expectation of a small DMI arising at the $\mathrm{Co} / \mathrm{Au}$ interface $[21,22]$. Gold therefore serves as a textbook element giving the opportunity to study the effect of controlled broken symmetry on either side of the ferromagnet and its importance for the various effects arising at these interfaces.

\section{EPILAYER GROWTH AND CHARACTERIZATION}

The trilayers of $\operatorname{Pt}(3 \mathrm{~nm}) / \mathrm{Co}(0.6 \mathrm{~nm}) / \mathrm{Au}_{x} \mathrm{Pt}_{1-x}(3 \mathrm{~nm})$, in which $0 \leqslant x \leqslant 1$, were prepared by sputtering at high temperatures, as described in Ref. [18]. The seed Pt layer was sputtered directly on a C-plane sapphire substrate at $500{ }^{\circ} \mathrm{C}$ followed by $\mathrm{Co}$ at $100^{\circ} \mathrm{C}$. The $\mathrm{Au}_{x} \mathrm{Pt}_{1-x}$ layer was grown by co-sputtering from $\mathrm{Pt}$ and $\mathrm{Au}$ targets at $100{ }^{\circ} \mathrm{C}$ in a way that the sputtering powers were adjusted to keep the rate $\sim 1$ $\AA /$ s. The multilayers were capped with $\sim 2 \mathrm{~nm}$ of Ta, which is polycrystalline.

The quality of these epilayers was confirmed by $\mathrm{x}$-ray diffraction (XRD) and transmission electron microscopy (TEM). The XRD spectra for $x=0,0.5$, and 1 , shown in Fig. 1(a), are consistent with the previous study [18]. In each case, a broad principal peak (due to the extreme thinness of the layers) is found close to $\theta=40^{\circ}$, surrounded by satellite Pendellösung fringes. This peak is very close to bulk $\mathrm{Pt}$ (111) Bragg peak at $\theta=39.74^{\circ}$, showing the matching of the multilayer structure with the substrate. The appearance of Pendellösung fringes in the XRD of a multilayer emphasizes the uniformity of the strain in the layers and smoothness of the interfaces $[23,24]$. The peak moves to slightly lower angles as $\mathrm{Au}$ is introduced into the upper layer, as might be expected from a Vegard law-type behavior.

Cross-sections of the three samples are shown as dark field high-resolution transmission electron microscopy (HRTEM) images in Fig. 1(b). Lattice fringes are visible extending coherently across all three layers, emphasising the epitaxial nature of the samples. Energy dispersive x-ray analyses of each of the three samples are shown in Fig. 1(c), showing distinct layers. The lattice spacing in the vertical direction, $d_{111}$, can be evaluated using the position of the main peak in the XRD pattern and Bragg's law, or by averaging the lattice spacings in the cross-sectional TEM images. Comparing evaluated values from both measurements, there is just about $2 \%$ difference in $d_{111}$ between them (within error bars), which is a good agreement.

The contrast in TEM images is related to the atomic number inducing a darker appearance for the Co layer in comparison with $\mathrm{Pt}$ and $\mathrm{Au}$. Investigating the changes of greyness in the vertical direction of images results in Co thicknesses of $1.03,1.26$, and $1.17 \mathrm{~nm}$ for $x=1,0.5$, and 0 , respectively.

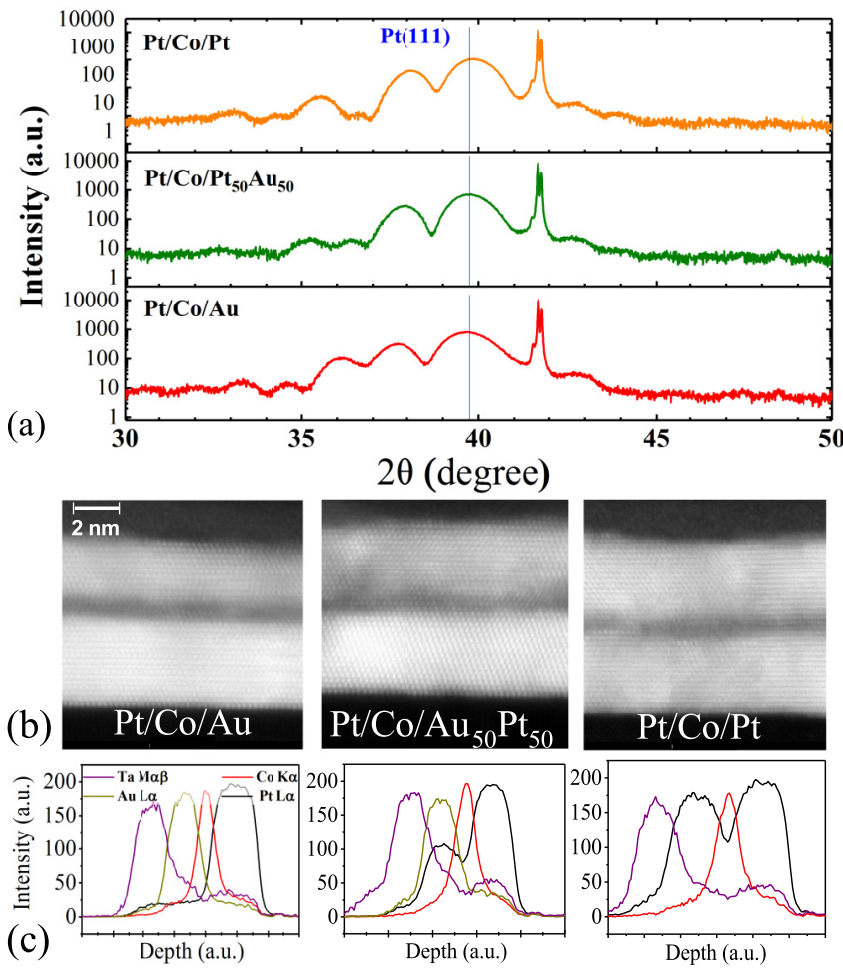

FIG. 1. Structural characterization of $\mathrm{Pt} / \mathrm{Co} / \mathrm{Au}_{x} \mathrm{Pt}_{1-x}$ trilayers. (a) X-ray diffraction spectra confirming the fcc (111) orientation of the samples. (b) Dark field HRTEM cross-section images. (c) EDX spectra showing distinguishable layers with possible intermixing at the interfaces.

(The thickness was measured for stripes of $\sim 0.4 \mathrm{~nm}$ width across the entire image, and then the average was calculated.) This is much higher than the nominal thickness of $0.6 \mathrm{~nm}$. In fact, this is expected as TEM shows the additive effect of all atomic layers in the depth of FIB-cut lamella (which is usually $\sim 50 \mathrm{~nm}$ ). So, when the electron beam goes through the TEM specimen, the thickness fluctuations of the thin layer will be added to the actual thickness resulting in the overestimation of thickness. It has also been reported previously than evaluating thickness from TEM cross-sections might result in overestimated thicknesses, sometimes even two times higher than the real value [25]. Hence x-ray reflectivity patterns of samples were fitted using the GENX package [26], which yielded thicknesses very close to the nominal ones for the Co layers. Considering all these facts, the nominal thickness of $0.6 \mathrm{~nm}$ was taken to be a good estimate of the true thickness value for further analysis of parameters describing the samples.

All the films showed square magneto-optical Kerr effect (MOKE) hysteresis loops as a function of perpendicular magnetic field, confirming that the PMA generated at the Coheavy metal interfaces is strong enough to overcome the shape anisotropy [Fig. 2(a)]. Superconducting quantum interference device vibrating sample magnetometry (SQUID-VSM) was used to measure the anisotropy field $H_{\mathrm{K}}$, inferred from the inplane saturation field, plotted in Fig. 2(b), and the saturation magnetization $M_{\mathrm{s}}$ shown in Fig. 2(c). To calculate $M_{\mathrm{s}}$, it was assumed that all the measured moment is confined in the Co layer of thickness $t$. However, due to the proximity 

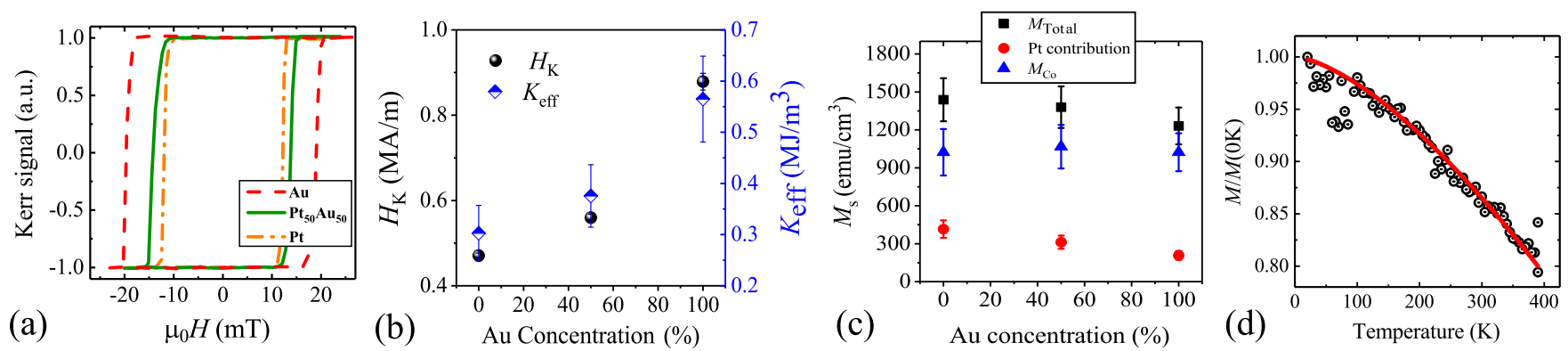

FIG. 2. Magnetic characterization of $\mathrm{Pt} / \mathrm{Co} / \mathrm{Au}_{x} \mathrm{Pt}_{1-x}$ trilayers. (a) Polar MOKE loops. (b) Anisotropy field, $H_{\mathrm{K}}$, and effective anisotropy energy, $K_{\text {eff }}$ both increase with Au concentration. (c) Saturation magnetization $M_{\mathrm{s}}$ supposing that all the magnetic moment is confined in the Co layer (black dots), extracted proximity-induced magnetism in Pt layers (red) and corrected Co layer values $M_{\mathrm{Co}}$ (blue). (d) Variation of relative saturation magnetization with temperature for $\mathrm{Pt} / \mathrm{Co} / \mathrm{Pt}$. The solid red line is a fit of the Bloch law.

effects, induced magnetic moments also exist in the Pt layers. The induced moment in Pt can be estimated by assuming a negligible proximity effect in $\mathrm{Au}$ [20], and so the difference in the moment between the $x=0$ and $x=1$ films corresponds to the induced moment in a single Pt layer. This is summarized in Fig. 2(c), which reveals the contribution of the magnetization in the $\mathrm{Pt}$ to the constant magnetization of the Co layer $(1.0 \pm 0.1 \mathrm{MA} / \mathrm{m})$. This is comparable to the previously published values for $\mathrm{Co} / \mathrm{Pt}$ multilayers with the same nominal thickness of cobalt [16,27-31]. On the other hand, if we consider thicknesses deduced from the TEM measurements, the saturation magnetization of mulilayers would be about $0.6-0.7 \mathrm{MA} / \mathrm{m}$, which is unusually low for supposedly $1 \mathrm{~nm}$ of Co [27,32]. This also emphasizes the unreliability of thickness estimations from TEM cross-sections. Following this analysis, the effective anisotropy can be calculated by $K_{\text {eff }}=\frac{1}{2} \mu_{0} M_{\mathrm{Co}} H_{\mathrm{K}}$, which is also plotted in Fig. 2(b), which rises markedly with Au content $x$. The values, along with the other magnetic data for each sample, are given in Table I. The temperature dependence of $M_{\mathrm{Co}}$ between 5 and $400 \mathrm{~K}$ for the $x=0$ sample shown in Fig. 2(d) was fitted with the Bloch law [33],

$$
\frac{M_{\mathrm{Co}(T)}}{M_{0}}=1-c\left(\frac{k_{\mathrm{B}} T}{J}\right)^{\frac{3}{2}}
$$

where $M_{0}$ is the magnetization at $0 \mathrm{~K}, c=0.0294$ for an fcc lattice, $k_{\mathrm{B}}$ is the Boltzmann constant, and $J$ is the exchange integral. Knowing $J$, one can obtain the exchange stiffness as $A=Q J S^{2} / a$, with $Q=4$ as the number of atoms per unit cell for fcc, $S$ the spin quantum number, and $a=0.355 \mathrm{~nm}$ the lattice constant. The value we obtain is $A=17 \pm 1 \mathrm{pJ} / \mathrm{m}$. This reduction in $A$ with respect to the bulk value for Co of $30 \mathrm{pJ} / \mathrm{m}$ is in good agreement with our previous findings for Co layers of this thickness [34].

\section{DOMAIN WALL CHIRALITY}

Ferromagnet-heavy metal interfaces also exhibit interfacial DMI [4], which enforces the Néel form of the DW profile with a fixed chirality $[6,10]$. Here, we have studied this property using the asymmetric bubble expansion method $[16,35]$. A bubble domain is nucleated and then expanded by an out-of-plane applied field $H_{z}$, whilst $H_{\mathrm{DMI}}$, the effective field induced at the DW by the DMI [10] is enhanced or compensated by an in-plane applied field $H_{x}$, resulting in different creep-regime velocities for walls on either side of the bubble moving with or against the in-plane field [35,36]. The effective micromagnetic DMI strength is given by $D_{\text {eff }}=$ $\mu_{0} H_{\mathrm{DMI}} M_{\mathrm{Co}} \Delta$, where $\Delta$ is the DW width. To measure $H_{\mathrm{DMI}}$ we have used Kerr microscopy with a two-coil setup to apply $H_{z}$ and $H_{x}$ independently. We measure the DW creep velocity as a function of $H_{x}$ by pulsing $H_{z}$ with a fixed amplitude. The DW motion at low magnetic fields follows the creep law [30], and its velocity can be expressed as

$$
v=v_{0} \exp \left(\zeta H_{z}^{-\frac{1}{4}}\right)
$$

TABLE I. Magnetic properties of epitaxial $\mathrm{Pt} / \mathrm{Co} / \mathrm{Au}_{x} \mathrm{Pt}_{1-x}$ for the three different values of $\mathrm{Au}$ concentration $x$ studied here. These are saturation magnetization $M_{\mathrm{CO}}$, uniaxial anisotropy constant $K_{\mathrm{u}}=K_{\text {eff }}+\frac{1}{2} \mu_{0} M_{\mathrm{Co}}^{2}$, DW width $\Delta=\sqrt{A / K_{\text {eff }}}$, Gilbert damping constant from creep and depinning fits $\alpha_{\text {exp }}$, Walker breakdown field $\mu_{0} H_{\mathrm{W}}$ calculated using DW mobility, Gilbert damping constant from micromagnetics $\alpha_{\mu \mathrm{M}}$, depinning field $H_{\mathrm{d}}$ obtained from fitting with the creep law, thickness fluctuation $\delta$ used in the micromagnetics simulations, correlation length of the disorder $\xi$, strength of the pinning disorder $f_{\text {pin }} / \xi$, and effective DMI constant $D_{\text {eff }}$, as well as the inferred handedness of the

\begin{tabular}{|c|c|c|c|c|c|c|c|c|c|c|c|c|}
\hline$x$ & $\begin{array}{c}M_{\mathrm{Co}} \\
\mathrm{MA} / \mathrm{m}\end{array}$ & $\begin{array}{c}K_{\mathrm{u}} \\
\mathrm{MJ} / \mathrm{m}^{3}\end{array}$ & $\begin{array}{c}\Delta \\
\mathrm{nm}\end{array}$ & $\alpha_{\exp }$ & $\begin{array}{c}\mu_{0} H_{\mathrm{W}} \\
\mathrm{mT}\end{array}$ & $\alpha_{\mu \mathrm{M}}$ & $\begin{array}{c}\mu_{0} H_{\mathrm{d}} \\
\mathrm{mT}\end{array}$ & $\delta$ & $\begin{array}{c}\xi \\
\mathrm{nm}\end{array}$ & $\begin{array}{c}f_{\mathrm{pin}} / \xi \\
\mathrm{meV}\end{array}$ & $\underset{\mathrm{mJ} / \mathrm{m}^{2}}{D_{\text {eff }}}$ & Chirality \\
\hline & $1.0 \pm$ & \pm 0.2 & $5.5 \pm 0.9$ & 0 & 280 & 5 & $72 \pm 2$ & $9 \%$ & 3 & 0 & - & $\mathrm{Le}$ \\
\hline 0.5 & $1.1 \pm 0.2$ & $1.1 \pm 0.2$ & $7 \pm 1$ & $0.4 \pm 0.1$ & $49 \pm 3$ & $0.35 \pm$ & $56 \pm 2$ & $8.5 \%$ & $21 \pm 2$ & $330 \pm 30$ & $-0.35 \pm 0.09$ & Left \\
\hline & $1.0 \pm 0.2$ & $1.0 \pm 0.2$ & $8 \pm 1$ & $0.2 \pm 0.1$ & $11 \pm 1$ & $0.17 \pm 0.01$ & $39 \pm 2$ & $7 \%$ & $22 \pm 2$ & $240 \pm 20$ & $-0.07 \pm 0.07$ & None \\
\hline
\end{tabular}
chirality of the domain walls. 

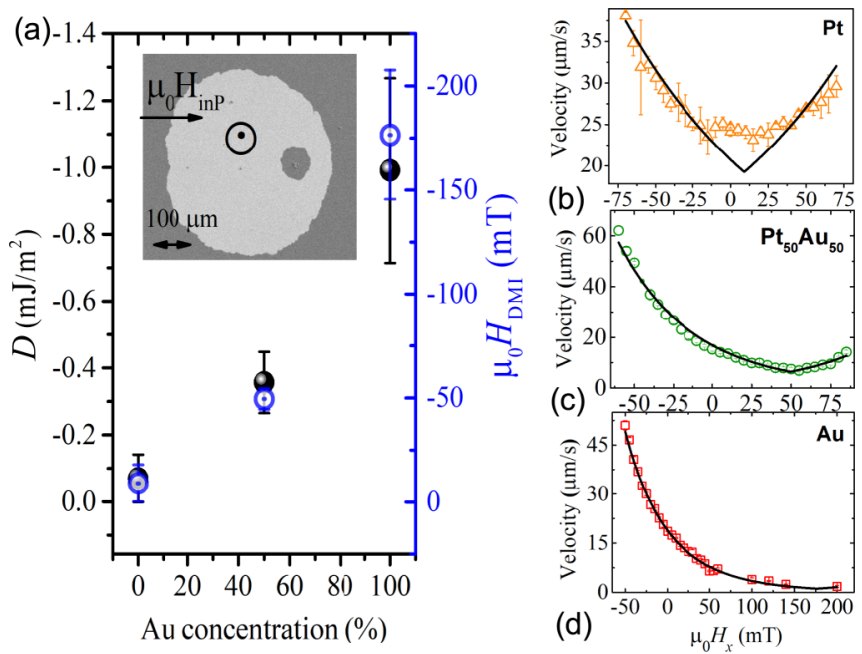

FIG. 3. Asymmetric bubble expansion measurements. (a) Effective DMI field $\mu_{0} H_{\text {DMI }}$ and effective DMI constant $D_{\text {eff }}$ as a function of Au concentration $x$ in $\mathrm{Pt} / \mathrm{Co}(6 \AA) / \mathrm{Au}_{x} \mathrm{Pt}_{1-x}$ epilayers. An example of a Kerr difference image of the bubble expansion in $\mathrm{Pt} / \mathrm{Co} / \mathrm{Au}$ is shown as an inset. The values plotted were derived from the data in (b)-(d), which show DW velocity as a function of in-plane magnetic field $\mu_{0} H_{x}$ in case of $\mathrm{Pt}, \mathrm{Pt}_{50} \mathrm{Au}_{50}$, and $\mathrm{Au}$ capping layers, respectively, for $\mu_{0} H_{z}=7,8$, and $10.5 \mathrm{mT}$.

where $v_{0}$ is the velocity prefactor, $H_{z}$ is out-of-plane driving field, and $\zeta$ is the scaling coefficient

$$
\zeta=\zeta_{0}\left[\frac{\sigma\left(H_{x}\right)}{\sigma(0)}\right]^{\frac{1}{4}}
$$

where $\zeta_{0}$ can be considered as a scaling constant for pinning properties independent of in-plane field. $\sigma$ is the Néel wall energy density [16,35], which contains a term proportional to $\left|H_{x}+H_{\text {DMI }}\right|$. Hence the highest wall energy, and [through Eqs. (2) and (3)] minimum DW creep velocity $v$ corresponds to the point where $\mu_{0} H_{x}=-\mu_{0} H_{\mathrm{DMI}}$, i.e., the point at which an external field transforms the Néel wall to a Bloch wall [16].

We performed bubble expansion measurements on all three epilayers, the results are shown in Fig. 3. Measurements as a function of $H_{z}$ at given values of $H_{x}$ (not shown) yielded a $\ln v \propto H_{z}^{-1 / 4}$ scaling, confirming that measurements are in the creep regime. A typical Kerr difference micrograph of an expanded DW bubble in $\mathrm{Pt} / \mathrm{Co} / \mathrm{Au}$ film is displayed in the inset of Fig. 3(a), showing the asymmetric expansion. Examples of data sets for $v\left(H_{x}\right)$ for $\mathrm{Pt}, \mathrm{Pt}_{50} \mathrm{Au}_{50}$, and $\mathrm{Au}$ capping layers are shown in Figs. 3(b), 3(c) and 3(d), respectively. One can immediately see a shift of velocity minimum away from zero for in-plane field, indicating the presence of a DMI. The solid lines correspond to the fits of Eq. (2). The effective DMI field for each sample, determined from these fits, is plotted in Fig. 3(a) as a function of Au concentration $x$. It should be noted that the high uncertainty in the $D$ value for Au capped trilayer is due to not reaching the minimum velocity (i.e., the inversion symmetry axis of the curve), as a result of experimental limitations, and so our value is based just on the fit to the data that is shown. Negligible DMI in $\mathrm{Pt} / \mathrm{Co} / \mathrm{Pt}$ confirms that the system has a high degree of inversion symmetry for $x=0$, which is not a trivial result
$[16,29]$, since small differences in top and bottom interface structure can easily break inversion symmetry and lead to an appreciable DMI [17]. The negligible DMI in this case can thus be expected to lead to walls of Bloch form with no fixed chirality.

Also, Kim et al. stated that this assumption that the velocity minimum occurs at $-\mu_{0} H_{\mathrm{DMI}}$ only holds if the minimum velocity falls on the inversion symmetry axis of the velocity curve [37]. In other words, it holds if the asymmetric contribution to the velocity is negligible. In such a case, any contribution from chiral damping is negligible and DMI can be accounted for all the chiral behavior in the sample. Examination of symmetric and asymmetric contributions of our velocity curves showed that the latter is one order of magnitude smaller than the former, proving the dominance of the symmetric contribution and ruling out any chiral damping effect.

The effective DMI field and the strength of DMI constant $D_{\text {eff }}$ increase with $x$. This is expected since the trilayers are becoming more inversion asymmetric. From the symmetries of the applied fields we deduce that for $x>0$, the DMI enforces left-handed chirality of the Néel walls, which is consistent with ab initio calculations for Pt/Co interfaces [22].

\section{FIELD-DRIVEN DOMAIN WALL DYNAMICS}

In the previous section, we considered some aspects of the creep regime, where the applied field is small with respect to the depinning field and DW motion is thermally activated. For applied fields exceeding the depinning field, DW dynamics first goes through a so-called depinning transition and then moves in the viscous flow regime where temperature plays no role: the DW velocity is controlled by damping, parameterized by the Gilbert damping coefficient $\alpha$. We measured the DW velocity across both regimes by investigating bubble expansion driven by a pulsed out-of-plane applied field using a fast microcoil of diameter $1 \mathrm{~mm}$ that produced pulses with a $200 \mathrm{~ns}$ rise time. The DW velocity is then calculated by dividing the distance, which the DW covered during the pulse by the pulse duration. The velocities as a function of out-ofplane field $\mu_{0} H_{z}$ are shown in Fig. 4(a). The DW velocity for a specific value of $H_{z}$ is rising with the broken inversion symmetry of the trilayer. The measured velocities cover the thermally activated creep regime, the depinning transition, and the beginning of the appearance of the expected linear change of velocity in the flow regime. The data were fitted simultaneously with the universal creep and depinning regime functions [38]:

$$
v(H)=\left\{\begin{array}{ll}
v\left(H_{\mathrm{d}}\right) \exp \left(-\frac{\Delta E}{k_{\mathrm{B}} T}\right) & \text { creep } \\
\frac{v_{\mathrm{T}}\left(H_{\mathrm{d}}\right)}{x_{0}}\left(\frac{H_{z}-H_{\mathrm{d}}}{H_{\mathrm{d}}}\right)^{\beta} & \text { depinning }
\end{array},\right.
$$

where $v\left(H_{\mathrm{d}}\right)$ is the creep prefactor corresponding to the velocity at depinning, $T$ is the temperature, $k_{\mathrm{B}}$ is the Boltzmann constant, $H_{\mathrm{d}}$ is the depinning field, $\Delta E=$ $k_{\mathrm{B}} T_{\mathrm{d}}\left[\left(H_{z} / H_{\mathrm{d}}\right)^{-1 / 4}-1\right]$ is the pinning potential, $v_{T}=$ $v\left(H_{\mathrm{d}}\right)\left(T_{\mathrm{d}} / T\right)^{\psi}$ is the DW velocity in a disorder-free case, $x_{0}=0.65 \pm 0.04$ is a dimensionless constant [39], and $\psi=$ 0.15 and $\beta=0.25$ are depinning exponents [38]. Here we define the creep regime as $H \leqslant H_{\mathrm{d}}$ and the depinning regime 

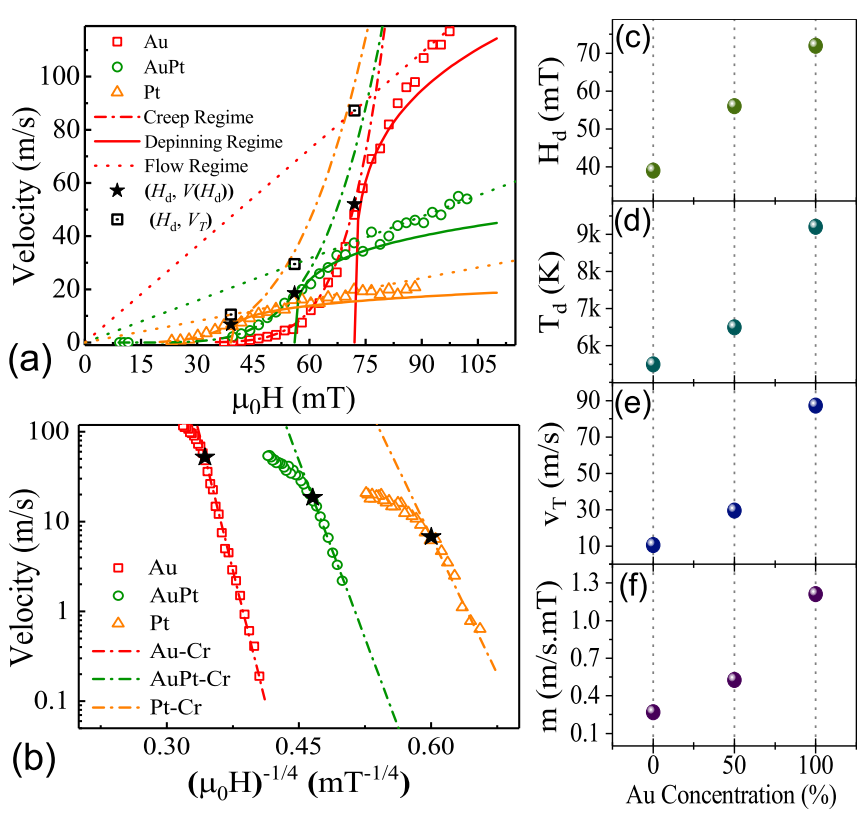

FIG. 4. OoP-only field-driven DW motion for $\mathrm{Pt} / \mathrm{Co} / \mathrm{Au}_{x} \mathrm{Pt}_{1-x}$ epilayers. (a) Fits to the data by universal models for creep (dasheddotted curve) and depinning regimes (solid curve) on a linear-linear plot. The dotted lines are linear fits to the viscous flow regime. (b) Semilogarithmic plot of the same velocity data as a function of $\left(\mu_{0} H\right)^{-1 / 4}$ to show fitting to the creep regime more clearly. Data are shifted to the right respectively for clarity of presentation. In each case, the solid stars show the depinning field $H_{\mathrm{d}}$. (c)-(f) Changes of derived parameters with concentration of gold $x$ : depinning field $H_{\mathrm{d}}$, depinning temperature $T_{\mathrm{d}}$, DW velocity in a film with pinning $v_{T}$, and DW mobility $m$, respectively.

as $H \gtrsim H_{\mathrm{d}}\left[1+\left(0.8\left(T_{\mathrm{d}} / T\right)^{-\psi}\right)^{1 / \beta}\right]$. The fitting results, which yield a good agreement with the acquired experimental data, are shown in Figs. 4(a) and 4(b). There is good agreement with both the creep law and with the depinning law for $H>H_{\mathrm{d}}$.

The depinning field $H_{\mathrm{d}}$ that separates the creep and depinning regimes of DW motion is marked on each curve in Fig. 4 by solid stars. The only nonuniversal (i.e., material and temperature dependent) parameters of the fits are the depinning field $H_{\mathrm{d}}$, depinning velocity $v_{\mathrm{T}}\left(H_{\mathrm{d}}\right)$, and depinning temperature $T_{\mathrm{d}}$. All these three parameters are increasing with the Au concentration in the system [Figs. 4(c)-4(e)]. Following Ref. [38], the material dependent parameters $H_{\mathrm{d}}$ and $T_{\mathrm{d}}$ can be used to estimate the variation with gold concentration of the coherence length $\left(\xi=\left[\left(k_{\mathrm{B}} T_{\mathrm{d}}\right)^{2} /\left(2 M_{\mathrm{s}} H_{\mathrm{d}} \sigma t^{2}\right)\right]^{1 / 3}\right)$, and the pinning strength $\left(f_{\text {pin }} \xi=\left[\left(k_{\mathrm{B}} T_{\mathrm{d}}\right)^{3} /(\sigma \xi t)\right]^{1 / 2}\right)$. The results are reported in Table I. It can be seen there that the correlation length of the disorder is almost $\operatorname{constant}(\xi=23 \pm 2 \mathrm{~nm})$, which suggests that the typical length scale of the variations of the pinning potential (i.e., of the fluctuation of DW energy) is independent of the gold concentration. Moreover, the increase of the pinning strength suggests that fluctuations of DW energy are larger at the CoAu interface than at the $\mathrm{CoPt}$ interface. This is compatible with a smearing of DW energy fluctuations at the CoPt interface due to intermixing, which do not exist at the CoAu interface since $\mathrm{Co}$ and $\mathrm{Au}$ do not form any alloy [40].
In each case the DW enters the flow regime, where $v \propto H_{z}$, at high fields. As stated before, $v_{T}$ is assumed to be the velocity of DW in the absence of pinning, hence the DW mobility will be $m=\mu_{0}^{-1} v_{T} / H_{\mathrm{d}}$. Using this calculated $m$ [Fig. 4(f)], a line can be drawn with this slope, going through origin, shown in Fig. 4(a) as a dotted line. Nontrivially, the line falls onto the experimental data points at high fields where $v \propto H_{z}$. This is interesting, as the calculated DW mobility (that is a flow regime related parameter) is derived from creep and depinning fits to the experimental data, which are measured with magnetic fields much lower than what viscous flow motion needs. In other words, we could learn about the flow regime and its parameters without a need to reach high, flow-related fields.

The standard one-dimensional (1D) model description of DW dynamics [10], which describes the DW motion in terms of its position $q$ and internal spin configuration $\phi$, predicts that the DW mobility $m$ in the flow regime depends on Gilbert damping $\alpha$ as follows:

$$
m= \begin{cases}\gamma \Delta / \alpha & \text { Steady flow } \\ \gamma \Delta /\left(\alpha+\alpha^{-1}\right) & \text { Precessional flow }\end{cases}
$$

where $\gamma$ is the gyromagnetic ratio [30]. The Gilbert damping for the three layers, $\alpha_{\exp }$, determined from these dashed lines is given in Table I. Equation (5), which relates the DW mobility to $\alpha_{\exp }$, only gives a solution in the case of precessional flow, the trilayers capped with Pt and AuPt, whilst yielding an only steady flow solution for the epilayer where $x=1$.

The Walker field, which separates the steady and precessional flow regimes, can be estimated by using relation [10]

$$
H_{\mathrm{W}}=\alpha_{\mathrm{exp}} \sin \Phi_{\mathrm{W}}\left(H_{\mathrm{DMI}}-H_{\mathrm{demag}} \cos \Phi_{\mathrm{W}}\right),
$$

where $H_{\text {demag }}$ is the DW demagnetizing field, and $\cos \Phi_{\mathrm{W}}=$ $\left(\left[H_{\text {DMI }} / H_{\text {demag }}\right]-\sqrt{\left[H_{\text {DMI }} / H_{\text {demag }}\right]^{2}+8}\right) / 4$. The values we obtain for $H_{\mathrm{W}}$ are also given in Table I. The Walker field increases markedly with $x$, due to the stronger DMI as the inversion asymmetry is progressively broken. It can be seen that knowledge of the DMI field and Gilbert damping are needed to determine the regime of the DW dynamics with respect to the Walker field. Nevertheless, comparing these values with $H_{\mathrm{d}}$, we can see that our results are self-consistent, confirming which flow regime each sample enters on exceeding the depinning field.

\section{MICROMAGNETIC SIMULATION}

The Gilbert damping values $\alpha_{\text {exp }}$ obtained from Eq. (5) are unexpectedly large in the $\mathrm{Pt} / \mathrm{Co} / \mathrm{Au}$ sample [30], suggesting that such a description, based on a 1D model, might not always be applicable in these systems. This could be due to a strong effect of disorder, since the 1D model usually assumes DW motion in perfectly homogeneous conditions.

In order to test this conjecture, we performed numerical micromagnetic simulations using the MUMAX ${ }^{3}$ package [41]. We used the experimentally measured magnetic parameters ( $A, K_{\mathrm{u}}, M_{\mathrm{Co}}$, and $\left.D_{\text {eff }}\right)$ and implemented disorder by means of Voronoi tessellation [see Fig. 5(a)] of the sort usually used to represent a grain structure $[42,43]$. Since our trilayers are epitaxial they do not have a grain structure per se, but 

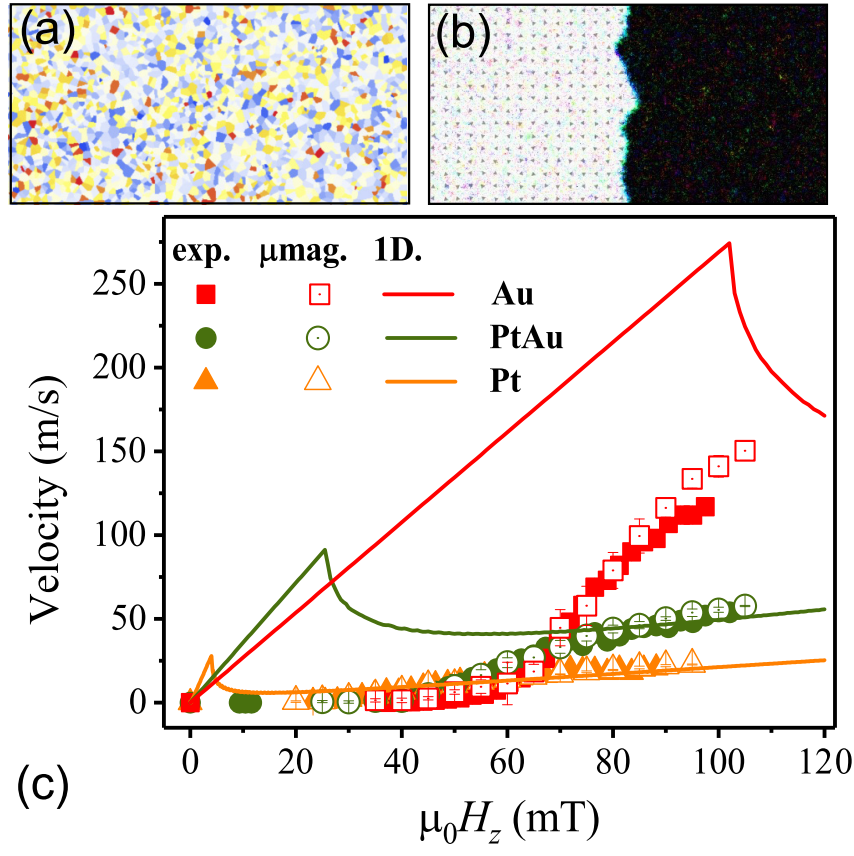

FIG. 5. Field-induced DW motion in $\mathrm{Pt} / \mathrm{Co} / \mathrm{Au}_{x} \mathrm{Pt}_{1-x}$ epilayers. (a) and (b) Visualizations of the micromagnetic simulations for $\mathrm{Pt} / \mathrm{Co} / \mathrm{Au}$ with $\delta=8.5 \%, \alpha=0.35$ at $t=0$ ns. (c) Field-dependent velocity curves along with the simulation from micromagnetic simulations and 1D model calculations. It is shown that 1D model calculations cannot reproduce the data while the micromagnetic simulations can follow change in velocity precisely.

there are magnetic layer thickness fluctuations, as revealed by the TEM cross-sections. In each region, the micromagnetic parameters were changed as expected from a random thickness fluctuations that affects $K_{\mathrm{u}}, D_{\text {eff }}$, and $M_{\text {Co }}$ [44-46]. The standard deviation of this fluctuation is given by the parameter $\delta$. The simulated sample is discretized in rectangular cells of $(2 \times 2 \times t) \mathrm{nm}^{3}$, where $t$ is the thickness of the sample. We consider a region of $1.0 \mu \mathrm{m} \times 0.5 \mu \mathrm{m}$, with periodic boundary condition along the $y$ direction. The DW is relaxed at the center as shown in Fig. 5(b). The "grain" size is set to $15 \mathrm{~nm}$, which is a scale consistent with our TEM analysis here, and close to the values of $\xi$ given in Table I. We simulate the DW dynamics for $50 \mathrm{~ns}$, in order to reach a stationary state in which the calculated DW velocity is not influenced by the simulation time. We performed different simulations combining different values of $\alpha$ and $\delta$. As all the experimental measurements were done at room temperature, the simulation temperature was set to $T=300 \mathrm{~K}$, accounted for by a thermal field of the form given by Brown [41,47]. The best fits are shown in Fig. 5(c) for the three samples. The description of the data with this approach is excellent. The corresponding best fitting parameters $\left(\alpha_{\mu \mathrm{M}}\right.$ and $\left.\delta\right)$ are reported in Table I. The larger $\delta$ we had to introduce for the samples doped with Au reflects the higher values of $f_{\text {pin }}$ due to the more abrupt interface between $\mathrm{Co}$ and $\mathrm{Au}$, as previously commented on.

In every case, $\alpha_{\mu \mathrm{M}}<\alpha_{\text {exp }}$, although the largest difference is for $x=1$. That damping parameter for $\mathrm{Pt} / \mathrm{Co} / \mathrm{Au}$ of is in reasonable agreement with the values reported for typical
$\mathrm{Pt} / \mathrm{Co}$ systems $\alpha \approx 0.3$ [30]. For Pt/Co/PtAu and $\mathrm{Pt} / \mathrm{Co} / \mathrm{Pt}$, the damping parameters are slightly lower but just within an error bar of $\alpha_{\text {exp }}$. We calculated the prediction of the 1D model for $\alpha_{\mu \mathrm{M}}$, also shown Fig. 5(c) as solid lines, which do not coincide with the data at all. The failure of the 1D model can be easily understood if we look at the depinning fields obtained from the creep fits. When $H_{z} \approx H_{\mathrm{d}}$ for $H_{\mathrm{d}}<H_{\mathrm{W}}$, as is the case for $\mathrm{Pt} / \mathrm{Co} / \mathrm{Au}$, disorder has a strong effect on the DW dynamics, which can be reproduced successfully only with full micromagnetic simulations. On the other hand, if $H_{z} \gg$ $H_{\mathrm{d}}>H_{\mathrm{W}}$, disorder has a smaller effect on the DW dynamics and the predictions of the 1D model are in agreement with micromagnetic calculations in that field regime, as is the case for $\mathrm{Pt} / \mathrm{Co} / \mathrm{Pt}$ and $\mathrm{Pt} / \mathrm{Co} / \mathrm{PtAu}$. Not taking into account the DW's internal dynamics (such as the presence and collision of Bloch lines) can only lead to an upper limit estimation for damping [46], due to the fact that all dissipation mechanisms are, implicitly, attributed to damping in that case [48].

The larger damping in the more strongly Au-doped samples could be due to a larger spin pumping [49] at the $\mathrm{Co} / \mathrm{Au}$ interface. An increased spin Hall effect was, indeed, recently observed in Pt/Au/Co stacks [50] and attributed to a larger spin transmission at the $\mathrm{Au} / \mathrm{Co}$ interface, which could also affect the spin pumping mechanism. However, it is unlikely that it could explain alone the large difference with the $\mathrm{Pt} / \mathrm{Co} / \mathrm{Pt}$ sample. Moreover, similar spin mixing conductances were observed in $\mathrm{Pt} / \mathrm{Co}$ and $\mathrm{Au} / \mathrm{Co}$ by FMR [51]. Nonlocal damping (dependent on the spin configuration) [52-54] could also play an important role for narrow DWs and could lead to an increased effective Gilbert damping. ${ }^{1}$ Hankiewicz et al., in particular, showed that nonlocal damping increases with the disorder scattering rate and is strongly enhanced by electron-electron interactions for weak spin polarization [52], as could occur at the $\mathrm{Co} / \mathrm{Au}$ interface or in case of intermixing. Nonlocal effects will also be stronger for the larger magnetization gradients around the narrower walls found for trilayers containing more Au. Also, it was reported that for $\mathrm{Au} / \mathrm{Co}$ interfaces the very interfacial atomic layers are playing the most important role in damping of the system, while for $\mathrm{Pt} / \mathrm{Co}$ bilayers the effect can be extended to a few atomic layers [55]. So the observed higher thickness fluctuations (i.e., interface roughness) and probable intermixing of layers for $\mathrm{Pt} / \mathrm{Co} / \mathrm{Au}$ trilayers of this study can affect the effective damping.

\section{CONCLUSIONS}

We have prepared and studied a series of $\mathrm{Pt} / \mathrm{Co} / \mathrm{Pt}_{1-x} \mathrm{Au}_{x}$ epitaxial trilayers with a well-defined crystallographic structure and controlled levels of proximity magnetism and inversion symmetry breaking. We have determined their static magnetic properties and the field-driven domain wall dynamics that they support. Our main findings are as follows. Comparing the measured moments of our samples allows us to estimate the degree of proximity magnetization in $\mathrm{Pt}$,

\footnotetext{
${ }^{1}$ The inclusion of the nonlocal damping would imply a modification of the LLG equation [52,53]. Thus the value fitted with the standard LLG must be seen as an effective damping, which averages out these inhomogeneous contributions.
} 
which is about one-third of the total moment in a $\mathrm{Pt} / \mathrm{Co}(0.6$ $\mathrm{nm}) / \mathrm{Pt}$ trilayer. Adding $\mathrm{Au}$ increases the PMA, and also breaks the inversion symmetry and increases the overall DMI. This DMI leads to homochiral left-handed Néel domain walls, as predicted by theory for $\mathrm{Pt} / \mathrm{Co}$ [22], suggesting that the DMI arising from the Au interface is weak, mirroring findings for the spin-orbit torque [15]. Adding Au also increases the depinning field and temperature for these walls, which is consistent with a more abrupt magnetic interface in the absence of proximity magnetism. The field-driven domain wall dynamics, including the crossover from creep to viscous flow, can be described well by a micromagnetic model that takes appropriate account of the disorder in the sample whereas a simple 1D model fails to do so. This micromagnetic modeling reveals lower values of Gilbert damping than the 1D case, due to the proper treatment of pinning, although still rising up to $\alpha \approx 0.3$ as $\mathrm{Au}$ is added. The rise is likely to be due to higher interface transparency leading to increased spin pumping or to higher levels of nonlocal damping. The overall approach to the study of these trilayers used here could be also extended to studying the physics of skyrmions [56,57], which contain the same physics as the chiral walls although expanded into two dimensions.

\section{ACKNOWLEDGMENTS}

This work was supported by the EU via WALL network (Grant No. FP7-PEOPLE-2013-ITN 608031), the UK EPSRC (Grant No. EP/I011668/1), and the Agence Nationale de la Recherche (France) under Contract No. ANR14-CE26-0012 (Ultrasky). The work by E. M. was supported by Projects MAT2014-52477-C5-4-P and MAT201787072- C4-1-P from the Spanish government, and Project No. SA090U16 from the Junta de Castilla y Leon. The data associated with this paper are publicly available from the University of Leeds Data Repository at the following URL: https://doi.org/10.5518/471.
[1] A. Hoffmann and S. D. Bader, Opportunities at the Frontiers of Spintronics, Phys. Rev. Appl. 4, 047001 (2015).

[2] W. J. M. de Jonge, P. J. H. Bloemen, and F. J. A. den Broeder, Experimental Investigations of Magnetic Anisotropy (Springer, Berlin, 1994), Chap. 2.3.

[3] W. J. Antel, M. M. Schwickert, T. Lin, W. L. O'Brien, and G. R. Harp, Induced ferromagnetism and anisotropy of Pt layers in Fe/Pt(001) multilayers, Phys. Rev. B 60, 12933 (1999).

[4] A. Fert, Magnetic and transport properties of metallic multilayers, in Metallic Multilayers, edited by A. Chamberod and J. Hillairet, Materials Science Forum Vol. 59 (1991), pp. 439-480.

[5] G. Chen, J. Zhu, A. Quesada, J. Li, A. T. N'Diaye, Y. Huo, T. P. Ma, Y. Chen, H. Y. Kwon, C. Won, Z. Q. Qiu, A. K. Schmid, and Y. Z. Wu, Novel Chiral Magnetic Domain Wall Structure in $\mathrm{Fe} / \mathrm{Ni} / \mathrm{Cu}$ (001) Films, Phys. Rev. Lett. 110, 177204 (2013).

[6] M.-J. Benitez, A. Hrabec, A. P. Mihai, T. A. Moore, G. Burnell, D. McGrouther, C. H. Marrows, and S. McVitie, Magnetic microscopy of topologically protected homochiral domain walls in an ultrathin perpendicularly magnetized Co film, Nat. Commun. 6, 8957 (2015).

[7] J.-P. Tetienne, T. Hingant, L. J. Martínez, S. Rohart, A. Thiaville, L. Herrera Diez, K. Garcia, J.-P. Adam, J.-V. Kim, J.-F. Roch, I. M. Miron, G. Gaudin, L. Vila, B. Ocker, D. Ravelosona, and V. Jacques, The nature of domain walls in ultrathin ferromagnets revealed by scanning nanomagnetometry, Nat. Commun. 6, 6733 (2015).

[8] Y. Tserkovnyak, A. Brataas, and G. E. W. Bauer, Enhanced Gilbert Damping in Thin Ferromagnetic Films, Phys. Rev. Lett. 88, 117601 (2002).

[9] A. Hubert and R. Schäfer, Magnetic Domains - The Analysis of Magnetic Microstructures (Springer, Berlin, 1998).

[10] A. Thiaville, S. Rohart, É. Jué, V. Cros, and A. Fert, Dynamics of Dzyaloshinskii domain walls in ultrathin magnetic films, Europhys. Lett. 100, 57002 (2012).

[11] S. S. P. Parkin and S.-H. Yang, Memory on the racetrack, Nat. Nanotechnol. 10, 195 (2015).
[12] A. Manchon, Spin-orbitronics: A new moment for Berry, Nat. Phys. 10, 340 (2014).

[13] A. V. Khvalkovskiy, V. Cros, D. Apalkov, V. Nikitin, M. Krounbi, K. A. Zvezdin, A. Anane, J. Grollier, and A. Fert, Matching domain-wall configuration and spin-orbit torques for efficient domain-wall motion, Phys. Rev. B 87, 020402 (2013).

[14] S. Emori, U. Bauer, S.-M. Ahn, E. Martinez, and G.S.D. Beach, Current-driven dynamics of chiral ferromagnetic domain walls, Nat. Mater. 12, 611 (2013).

[15] K.-S. Ryu, S.-H. Yang, L. Thomas, and S. S. P. Parkin, Chiral spin torque arising from proximity-induced magnetization, Nat. Commun. 5, 3910 (2014).

[16] A. Hrabec, N. A. Porter, A. Wells, M. J. Benitez, G. Burnell, S. McVitie, D. McGrouther, T. A. Moore, and C. H. Marrows, Measuring and tailoring the Dzyaloshinskii-Moriya interaction in perpendicularly magnetized thin films, Phys. Rev. B 90, 020402 (2014).

[17] A. W. J. Wells, P. M. Shepley, C. H. Marrows, and T. A. Moore, Effect of interfacial intermixing on the Dzyaloshinskii-Moriya interaction in Pt/Co/Pt, Phys. Rev. B 95, 054428 (2017).

[18] A. P. Mihai, A. L. Whiteside, E. J. Canwell, C. H. Marrows, M.-J. Benitez, D. McGrouther, S. McVitie, S. McFadzean, and T. A. Moore, Effect of substrate temperature on the magnetic properties of epitaxial sputter-grown $\mathrm{Co} / \mathrm{Pt}$, Appl. Phys. Lett. 103, 262401 (2013).

[19] E. C. Stoner, Collective electron ferromagnetism, Proc. R. Soc. London A 165, 372 (1938).

[20] F. Wilhelm, M. Angelakeris, N. Jaouen, P. Poulopoulos, E. Th. Papaioannou, Ch. Mueller, P. Fumagalli, A. Rogalev, and N. K. Flevaris, Magnetic moment of $\mathrm{Au}$ at $\mathrm{Au} / \mathrm{Co}$ interfaces: A direct experimental determination, Phys. Rev. B 69, 220404 (2004).

[21] V. Kashid, T. Schena, B. Zimmermann, Y. Mokrousov, S. Blügel, V. Shah, and H. G. Salunke, Dzyaloshinskii-Moriya interaction and chiral magnetism in $3 d-5 d$ zigzag chains: Tightbinding model and ab initio calculations, Phys. Rev. B 90, 054412 (2014). 
[22] H. Yang, A. Thiaville, S. Rohart, A. Fert, and M. Chshiev, Anatomy of Dzyaloshinskii-Moriya Interaction at Co/Pt Interfaces, Phys. Rev. Lett. 115, 267210 (2015).

[23] K. Ploog, W. Stolz, and L. Tapfer, Effect of barrier configuration and interface quality on structural and electronic properties and MBE-grown $\mathrm{Al}_{x} \mathrm{Ga}_{1-x}$ As/GaAs, $\mathrm{Al}_{x} \mathrm{Ga}_{1-x} \mathrm{Sb} / \mathrm{GaSb}$ and $\mathrm{Al}_{x} \mathrm{In}_{1-x}$ As $/ \mathrm{Ga}_{x} \mathrm{In}_{1-x}$ As superlattices, in Thin Film Growth Techniques for Low-Dimensional Structures, edited by R. F. C. Farrow, S. S. P. Parkin, P. J. Dobson, J. H. Neave, and A. S. Arrott, Nato Science Series B Vol. 163 (Springer Science \& Business Media, 2013).

[24] B. K. Tanner, Characterization of Crystal Growth Defects by X-Ray Methods, Nato Science Series B (Springer US, 2013).

[25] M. S. Gabureac, D. A. MacLaren, H. Courtois, and C. H. Marrows, Long-ranged magnetic proximity effects in noble metaldoped cobalt probed with spin-dependent tunnelling, New J. Phys. 16, 043008 (2014).

[26] M. Björck and G. Andersson, GenX: an extensible X-ray reflectivity refinement program utilizing differential evolution, J. Appl. Crystallogr. 40, 1174 (2007).

[27] C.-J. Lin, G. L. Gorman, C. H. Lee, R. F. C. Farrow, E. E. Marinero, H. V. Do, H. Notarys, and C. J. Chien, Magnetic and structural properties of Co/Pt multilayers, J. Magn. Magn. Mater. 93, 194 (1991).

[28] S. Bandiera, R. C. Sousa, B. Rodmacq, and B. Dieny, Enhancement of perpendicular magnetic anisotropy through reduction of Co-Pt interdiffusion in $(\mathrm{Co} / \mathrm{Pt})$ multilayers, Appl. Phys. Lett. 100, 142410 (2012).

[29] R. Lavrijsen, D. M. F. Hartmann, A. van den Brink, Y. Yin, B. Barcones, R. A. Duine, M. A. Verheijen, H. J. M. Swagten, and B. Koopmans, Asymmetric magnetic bubble expansion under in-plane field in $\mathrm{Pt} / \mathrm{Co} / \mathrm{Pt}$ : effect of interface engineering, Phys. Rev. B 91, 104414 (2015).

[30] P. J. Metaxas, J. P. Jamet, A. Mougin, M. Cormier, J. Ferré, V. Baltz, B. Rodmacq, B. Dieny, and R. L. Stamps, Creep and Flow Regimes of Magnetic Domain-Wall Motion in Ultrathin $\mathrm{Pt} / \mathrm{Co} / \mathrm{Pt}$ Films with Perpendicular Anisotropy, Phys. Rev. Lett. 99, 217208 (2007).

[31] I. M. Miron, G. Gaudin, S. Auffret, B. Rodmacq, A. Schuhl, S. Pizzini, J. Vogel, and P. Gambardella, Current-driven spin torque induced by the Rashba effect in a ferromagnetic metal layer, Nat. Mater. 9, 230 (2010).

[32] M. Vaňatka, J.-C. Rojas-Sánchez, J. Vogel, M. Bonfim, M. Belmeguenai, Y. Roussigné, A. Stashkevich, A. Thiaville, and S. Pizzini, Velocity asymmetry of Dzyaloshinskii domain walls in the creep and flow regimes, J. Phys.: Condens. Matter 27, 326002 (2015).

[33] S. Chikazumi, Physics of Ferromagnetism, 2nd ed. (Oxford University Press, Oxford, 1997).

[34] P. M. Shepley, H. Tunnicliffe, K. Shahbazi, G. Burnell, and T. A. Moore, Magnetic properties, domain-wall creep motion, and the Dzyaloshinskii-Moriya interaction in $\mathrm{Pt} / \mathrm{Co} / \mathrm{Ir}$ thin films, Phys. Rev. B 97, 134417 (2018).

[35] S.-G. Je, D.-H. Kim, S.-C. Yoo, B.-C. Min, K.-J. Lee, and S.-B. Choe, Asymmetric magnetic domain-wall motion by the Dzyaloshinskii-Moriya interaction, Phys. Rev. B 88, 214401 (2013).

[36] Y. P. Kabanov, Y. L. Iunin, V. I. Nikitenko, A. J. Shapiro, R. D. Shull, L. Y. Zhu, and C. L. Chien, In-plane field effects on the dynamics of domain walls in ultrathin Co films with perpendicular anisotropy, IEEE Trans. Magn. 46, 2220 (2010).

[37] D.-Y. Kim, M.-H. Park, Y.-K. Park, J.-S. Kim, Y.-S. Nam, D.-H. Kim, S.-G. Je, H.-C. Choi, B.-C. Min, and S.-B. Choe, Chirality-induced antisymmetry in magnetic domain wall speed, NPG Asia Mater. 10, e464 (2018).

[38] V. Jeudy, R. Dìaz Pardo, W. Savero Torres, S. Bustingorry, and A. B. Kolton, Pinning of domain walls in thin ferromagnetic films, Phys. Rev. B 98, 054406 (2018).

[39] R. Diaz Pardo, W. Savero Torres, A. B. Kolton, S. Bustingorry, and V. Jeudy, Universal depinning transition of domain walls in ultrathin ferromagnets, Phys. Rev. B 95, 184434 (2017).

[40] T. B. Massalski, H. Okamoto, P. R. Subramanian, L. Kacprzak, and W. W. Scott, in Binary Alloy Phase Diagrams (American Society for Metals, Metals Park, OH, 1986), Vol. 1.

[41] A. Vansteenkiste, J. Leliaert, M. Dvornik, M. Helsen, F. GarciaSanchez, and B. Van Waeyenberge, The design and verification of MuMax3, AIP Adv. 4, 107133 (2014).

[42] J.-V. Kim and M.-W. Yoo, Current-driven skyrmion dynamics in disordered films, Appl. Phys. Lett. 110, 132404 (2017)

[43] K. Zeissler, M. Mruczkiewicz, S. Finizio, J. Raabe, P. M. Shepley, A. V. Sadovnikov, S. A. Nikitov, K. Fallon, S. McFadzean, S. McVitie, T. A. Moore, G. Burnell, and C. H. Marrows, Pinning and hysteresis in the field dependent diameter evolution of skyrmions in $\mathrm{Pt} / \mathrm{Co} / \mathrm{Ir}$ superlattice stacks, Sci. Rep. 7, 15125 (2017).

[44] A. Hrabec, J. Sampaio, M. Belmeguenai, I. Gross, R. Weil, S. M. Chérif, A. Stashkevich, V. Jacques, A. Thiaville, and S. Rohart, Current-induced skyrmion generation and dynamics in symmetric bilayers, Nat. Commun. 8, 15765 (2017).

[45] I. Gross, W. Akhtar, A. Hrabec, J. Sampaio, L. J. Martínez, S. Chouaieb, B. J. Shields, P. Maletinsky, A. Thiaville, S. Rohart, and V. Jacques, Skyrmion morphology in ultrathin magnetic films, Phys. Rev. Mater. 2, 024406 (2018).

[46] S. Moretti, M. Voto, and E. Martinez, Dynamical depinning of chiral domain walls, Phys. Rev. B 96, 054433 (2017).

[47] W. F. Brown, Thermal fluctuations of a single-domain particle, J. Appl. Phys. 34, 1319 (1963).

[48] Y. Yoshimura, K.-J. Kim, T. Taniguchi, T. Tono, K. Ueda, R. Hiramatsu, T. Moriyama, K. Yamada, Y. Nakatani, and T. Ono, Soliton-like magnetic domain wall motion induced by the interfacial Dzyaloshinskii-Moriya interaction, Nat. Phys. 12, 157 (2015).

[49] Y. Tserkovnyak, A. Brataas, and G. E. W. Bauer, Spin pumping and magnetization dynamics in metallic multilayers, Phys. Rev. B 66, 224403 (2002).

[50] M. Mann and G. S. D. Beach, Reduction of in-plane field required for spin-orbit torque magnetization reversal by insertion of $\mathrm{Au}$ spacer in $\mathrm{Pt} / \mathrm{Au} / \mathrm{Co} / \mathrm{Ni} / \mathrm{Co} / \mathrm{Ta}$, APL Mater. 5, 106104 (2017).

[51] B. B. Singh, S. K. Jena, and S. Bedanta, Study of spin pumping in co thin film vis-à-vis seed and capping layers using ferromagnetic resonance spectroscopy, J. Phys. D: Appl. Phys. 50, 345001 (2017).

[52] E. M. Hankiewicz, G. Vignale, and Y. Tserkovnyak, Inhomogeneous Gilbert damping from impurities and electron-electron interactions, Phys. Rev. B 78, 020404 (2008). 
[53] S. Zhang and S. S.-L. Zhang, Generalization of the LandauLifshitz-Gilbert Equation for Conducting Ferromagnets, Phys. Rev. Lett. 102, 086601 (2009).

[54] T. Weindler, H. G. Bauer, R. Islinger, B. Boehm, J.-Y. Chauleau, and C. H. Back, Magnetic Damping: Domain Wall Dynamics Versus Local Ferromagnetic Resonance, Phys. Rev. Lett. 113, 237204 (2014).
[55] E. Barati, M. Cinal, D. M. Edwards, and A. Umerski, Gilbert damping in magnetic layered systems, Phys. Rev. B 90, 014420 (2014).

[56] A. Fert, V. Cros, and J. Sampaio, Skyrmions on the track, Nat. Nanotechnol. 8, 152 (2013).

[57] N. Nagaosa and Y. Tokura, Topological properties and dynamics of magnetic skyrmions, Nat. Nanotechnol. 8, 899 (2013). 